\title{
Comunicação e percepção de risco: diferentes modos de comunicar, diferentes modos de partilhar a decisão clínica
}

Ricardo Rodrigues, ${ }^{1}$ Ana Rita Maria, ${ }^{2}$ Ana Bragança, ${ }^{3}$ Susana Simões, ${ }^{4}$ André Tomé, ${ }^{5}$ David Rodrigues, ${ }^{6}$ Daniel Pinto, ${ }^{7}$ Bruno Heleno ${ }^{8}$

\section{RESUMO}

Uma decisão clínica informada deriva de um diálogo, no qual ocorre a partilha de especificidades biopsicossociais, preferências e valores de uma pessoa com o seu médico. Este, por sua vez, partilha informações associadas a um risco epidemiológico ou de uma intervenção. O risco pode ser comunicado por palavras, números ou gráficos, sendo que o formato escolhido influencia as percepções e os comportamentos dos utentes. O formato nem sempre é compreendido e a incapacidade de raciocinar com informação numérica (inumeracia) constitui uma importante barreira a uma comunicação eficaz. Apesar disso, é possível minorar o impacto da inumeracia através de estratégias comunicacionais. As frequências naturais, a redução de risco absoluto e a utilização de pictogramas permitem uma percepção mais realista do risco e tornam-no mais inteligível, independentemente do grau de numeracia. Existe sempre algum grau de incerteza associado às estimativas de risco, mas o mais importante é ajudar o utente a lidar com a incerteza. Além disso, o risco não deve ser encarado num contexto populacional, mas antes personalizado, tendo em conta os factores pessoais que modulam os benefícios ou prejuízos associados às intervenções propostas. Independentemente das políticas populacionais existentes, uma decisão de saúde cabe sempre ao utente, pessoa livre e autónoma.

Palavras-chave: Risco, Comunicação em Saúde, Incerteza, Tomada de Decisões, Medicina Individualizada.

\section{COMUNICAÇÃO DE RISCO}

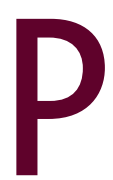

ode definir-se risco como a quantificação da ocorrência de um determinado evento prejudicial, neutro ou benéfico. ${ }^{1}$ No meio médico é geralmente entendido como a probabilidade de se desenvolver uma determinada doença, ${ }^{2}$ tendo-se ampliado o conceito para incluir os efeitos nocivos da intervenção médica (prevenção quaternária). ${ }^{3}$

1Médico Interno de Medicina Geral e Familiar, USF Conde de Oeiras 2Médica Interna de Medicina Geral e Familiar, USF Conde de Oeiras ${ }^{3}$ Médica Interna de Medicina Geral e Familiar, USF do Arco

${ }^{4}$ Estudante de Doutoramento em Ensino e Divulgação das Ciências, Faculdade de Ciências, Universidade do Porto

${ }^{5}$ Médico de Família, USF do Arco

${ }^{6}$ Departamento de Medicina Geral e Familiar, NOVA Medical School/Faculdade de Ciências Médicas, Universidade Nova de Lisboa

${ }^{7}$ Departamento de Medicina Geral e Familiar, NOVA Medical School/Faculdade de Ciências Médicas, Universidade Nova de Lisboa

${ }^{8}$ Médico de Família, Unidade de Investigação em Clínica Geral, Universidade de Copenhaga
A comunicação destes riscos tornou-se uma competência essencial para os médicos de família, tendo em vista a promoção de decisões clínicas partilhadas e informadas. De acordo com o modelo centrado no paciente, o utente é o elemento central na tomada de decisões de saúde. Primeiro, deverá adquirir conhecimentos sobre a sua doença e as opções terapêuticas disponíveis. Só então poderá tomar uma decisão esclarecida, de acordo com os seus valores e preferências. Neste sentido, os médicos de família devem saber: 1) as frequências das doenças e os efeitos das intervenções; 2) comunicar estas frequências e efeitos e 3) evocar preferências e atitudes.

Se realizada adequadamente, a comunicação de risco poderá ajudar a combater a ilusão da certeza: "os tratamentos só trazem benefícios, sem qualquer prejuízo"; "existe um e apenas um melhor tratamento"; "um teste diagnóstico é absolutamente certo". Estas formas de 
interpretar a realidade constituem um obstáculo mental para se tomar uma decisão esclarecida. Uma decisão é sempre uma escolha entre duas ou mais incertezas. ${ }^{1}$

Muitas vezes, os utentes e os médicos apresentam dificuldades em pesar os benefícios e prejuízos de diferentes opções terapêuticas. Além disso, a educação médica aborda de forma insuficiente as estratégias de comunicação e os modos de partilhar a decisão de saúde. O resultado é uma prática clínica que pode conduzir a decisões unilaterais ou pouco esclarecidas.

O objectivo deste artigo consiste em fornecer ferramentas que facilitem a comunicação de risco e ajudem a lidar com o seu grau de complexidade. Serão abordadas as diferentes formas de comunicar o risco (palavras, números ou gráficos) e a sua influência na percepção e na tomada de uma decisão pelo utente. Exploram-se, ainda, os temas da inumeracia, do efeito da personalização das mensagens e da comunicação da incerteza sobre os riscos. O artigo foca-se exclusivamente na comunicação de frequências e efeitos. Para ilustrar os aspectos teóricos mais relevantes recorrer-se-á a um diálogo ficcionado acerca do rastreio de cancro colo-rectal (CCR) no contexto de uma consulta de medicina geral e familiar. Os dados do diálogo baseiam-se na revisão sistemática mais recente sobre rastreio de CCR publicada pela colaboração Cochrane. ${ }^{4}$

O Sr. Silva vem à consulta da sua Médica de Família, Dra Prudência, para discutir o rastreio do CCR. Na semana anterior tinha conversado com o seu colega de trabalho sobre a necessidade de realizar este exame, tendo em conta que já tem 50 anos. Ficou a saber que a pesquisa de sangue oculto nas fezes (PSOF) detecta um cancro antes de se verificar qualquer sintoma, pelo que "só pode ser uma coisa boa!" Apesar disso, encara com algum cepticismo este rastreio, lembrando-se de um amigo seu, cujo resultado do exame foi positivo, revelando-se posteriormente um "falso alarme". O Sr. Silva pretende informar-se melhor acerca dos beneficios e prejuizos do rastreio através da PSOF, antes de tomar uma decisão.

Para o Sr. Silva são relevantes o risco de ter CCR, de morrer por CCR e de ter um falso positivo, se aceitar ser rastreado. De referir que o Sr. Silva não toma medicação, não apresenta queixas, nem tem antecedentes familiares ou pessoais relevantes para neoplasia intestinal.
A Dra Prudência realiza, desde há 20 anos, consultas centradas no paciente, pelo que se sente confortável em descobrir quais as preferências e atitudes dos seus utentes. Leu ainda recentemente um resumo da última revisão sistemáticat ${ }^{4}$ sobre rastreio de CCR. Contudo, apresenta algumas questões sobre a melhor forma de comunicar os riscos das doenças e os efeitos dos testes.

\section{COMPREENSÃO DE DADOS NUMÉRICOS NA DECISÃO CLÍNICA}

Frequentemente comunica-se o risco através de números. A interpretação desta informação é influenciada pela capacidade de compreender e utilizar informação matemática simples (numeracia), ${ }^{1}$ mas também pelo formato da informação transmitida (percentagens, frequências naturais, riscos absolutos e relativos, número necessário tratar).

Nem todas as pessoas são capazes de compreender e utilizar correctamente os números na tomada de uma decisão. ${ }^{2}$ Vários estudos documentam que muitos utentes têm dificuldade em compreender informação quantitativa em geral ${ }^{5}$ e que muitos profissionais de saúde cometem erros na interpretação de informação estatística, como por exemplo na compreensão de riscos relativos e valores preditivos. ${ }^{1-2,5}$ Com efeito, a educação e a numeracia associam-se, mas não constituem sinónimos. É necessário tomar em consideração que a dificuldade em pensar com números poderá não decorrer somente de uma formação insuficiente. Em parte, poderá derivar de representações que não correspondem ao modo de funcionamento intuitivo da nossa mente. $^{1}$

O desconforto provocado por sintomas (e.g., dor, dispneia), a tensão emocional e a curta duração da consulta comprometem a capacidade de raciocínio, mesmo em pessoas com boas capacidades numéricas. Quando a dificuldade da decisão ultrapassa a capacidade de raciocínio lógico, os utentes decidem, recorrendo a fontes emocionais em detrimento da informação providenciada. Isto sucede, por um lado, porque a informação emocional é mais fácil de processar. Por outro lado, a quantidade de informação pode ser demasiada para o tempo de consulta disponível. ${ }^{2}$ Deste modo, a inumeracia influencia as decisões de saúde, acabando por ter impacto na saúde a nível individual e populacional. 
Pelos motivos apresentados, compreende-se a relevância de discutir as melhores formas de apresentar a informação numérica. Boas escolhas podem minorar os efeitos da inumeracia.

\section{ENQUADRAMENTO}

A Dra Prudência consulta o quadro-resumo da revisão sistemática:em 1.000 pessoas, 20 desenvolvem CCR, sobrevivendo 12. Como transmitir esta frequência de mortalidade? Deverá dizer ao Sr. Silva que "12 pessoas em 20 sobrevivem a CCR" ou que "em 20 pessoas com CCR, morrem 8"? Ambas as frases fornecem a mesma informação. Mas qual delas é melhor compreendida?

$\mathrm{O}$ risco de desenvolver uma doença, bem como os prejuízos ou benefícios de uma intervenção, podem ser retratados de um modo positivo, como um ganho, ou de um modo negativo, como uma perda. Estas diferenças na forma de apresentar o risco são designadas de enquadramento. ${ }^{6}$ Distinguem-se dois tipos de enquadramento: atributos e efeitos.

$\mathrm{O}$ enquadramento de atributos permite retratar o risco associado a uma doença como algo positivo ou negativo. Como exemplo de enquadramento de atributos positivo temos " 12 pessoas em 20 sobrevivem a CCR" e, como exemplo de enquadramento de atributos negativo, "em 20 pessoas com CCR, morrem 8". O enquadramento de efeitos, por seu lado, possibilita a apresentação das consequências decorrentes de uma intervenção como um ganho (e.g., "a realização de PSOF aumenta a probabilidade de sobreviver a CCR") ou como uma perda (e.g., "a não realização de PSOF aumenta a probabilidade de morrer de CCR").

Uma revisão da Cochrane concluiu que as mensagens sobre atributos são melhor compreendidas quando apresentadas de modo negativo. Relativamente ao enquadramento de efeitos, não existem estudos sobre compreensão da mensagem. Contudo, verifica-se uma percepção de maior eficácia, em relação aos rastreios, utilizando o enquadramento de efeitos negativo. ${ }^{6} \mathrm{~A}$ melhor compreensão ou percepção de maior eficácia podem não se traduzir em alterações no comportamento. Ao contrário do que se pensava, a prova científica actualmente disponível, de qualidade baixa a moderada, sugere que o enquadramento de atributos e efeitos tem um efeito bastante reduzido ou mesmo nulo na tomada de decisão em saúde. ${ }^{6}$
Apesar de não se verificarem efeitos do enquadramento a nível do comportamento, desconhece-se se a percepção de uma maior eficácia determinará uma melhor resposta ao tratamento ou que efeitos terá do ponto de vista psicológico. A mesma informação, transmitida por palavras diferentes, é recebida de um modo também diferente. A utilização de antónimos, sinónimos ou de negação são aspectos ainda pouco investigados, mas poderão originar diferentes reacções no interlocutor.

\section{FORMATO}

\section{Números}

A Dra Prudência considera importante transmitir ao Sr. Silva o seu risco de desenvolver CCR. Decide apresentar esta informação utilizando enquadramento de atributos negativo:

Dra Prudência - Sr. Silva, antes de tomar uma decisão, tem de ter em conta que o seu risco de desenvolver CCR é de 0,02.

Sr. Silva - Ah... mas então isso é um risco muito baixo, ou estarei enganado?

Dra Prudência - Dizendo por outras palavras, o seu risco de desenvolver CCR é de $2 \%$.

Sr. Silva - Então, mas afinal o risco é de 2 ou de 0,02? Assim já parece um pouco maior.

Dra Prudência-São formas diferentes de dizer o mesmo. Melhor dizendo, em 1.000 pessoas como o senhor (entre os 50 e os 70 anos) que não fazem rastreio, 20 têm cancro do intestino.

A médica do Sr. Silva pretende transmitir-lhe o risco que este apresenta de desenvolver CCR. Este risco pode ser representado na forma de proporção (a proporção de pessoas que desenvolvem CCR é de 0,02 ), de percentagem (dois por cento das pessoas desenvolvem CCR) ou de frequência absoluta (em cada 1.000 pessoas, 20 desenvolverão CCR). ${ }^{4}$

Matematicamente não existe diferença em expressar a informação como proporção, percentagem ou frequência absoluta. No entanto, a compreensão, a percepção de risco e o comportamento são influenciados pela forma de representar o risco. ${ }^{7}$

As percentagens constituem uma forma de representação estatística que as pessoas poderão não saber calcular e consequentemente não compreender a sua 
relevância. ${ }^{1}$ Entendem-nas como abstractas e sentem que não se aplicam a elas próprias. As frequências naturais são percepcionadas como mais vívidas e pessoais. Por este motivo, muitos advogam a conversão de percentagens em frequências naturais. Numa revisão publicada pela Cochrane concluiu-se que as frequências naturais são melhor compreendidas que as percentagens. Parecem, ainda, constituir a melhor forma de comunicar risco de doença em contexto de rastreio ou testes diagnósticos. ${ }^{8}$

No entanto, é necessário ter cuidado com dois potenciais problemas que surgem com a utilização de frequências naturais: negligência do denominador e confusão do denominador. A negligência do denominador deriva do facto de a atenção se focar no número de eventos em detrimento do tamanho da população. Por exemplo, pode percepcionar-se o risco de desenvolver CCR de 2 em 100 como inferior a $20 \mathrm{em} \mathrm{1.000,} \mathrm{quando} \mathrm{na} \mathrm{rea-}$ lidade são riscos equivalentes. ${ }^{4,9}$ Confusão do denominador surge quando se comparam riscos com diferentes denominadores. Algumas pessoas têm maior probabilidade de focar-se no numerador e confundir-se ao não reconhecer, por exemplo, que o risco de morrer por CCR de 8 em 1.000 é menor que o risco de desenvolver CCR de 2 em 100. Deste modo, a informação deve ser sempre apresentada com os mesmos denominadores. O exemplo acima referido seria melhor compreendido se se comparasse o risco de desenvolver CCR de $20 \mathrm{em}$ 1.000 com o de morrer por CCR de 8 em 1.000. ${ }^{4,9}$
Dra Prudência-E se essas 1.000 pessoas fizessem rastreio, morria menos uma pessoa de cancro do intestino.

Sr. Silva - Menos uma pessoa em 1.000? Isso pareceme роисо...

Dra Prudência-Olhe que com o teste às fezes há 12,5\% menos mortes...

Sr. Silva - Então prefiro fazer o teste às fezes do que o rastreio.

Dra Prudência-Hmmm, acho que não me expliquei bem. Deixe-me lá tentar outra vez: é preciso rastrear 1.000 pessoas para que uma delas beneficie do rastreio.

Sr. Silva - Oh Doutora, já não percebo nada! É boa ideia fazer o teste às fezes ou não?

A variação de risco associada a determinada intervenção (a relação entre o risco final e o risco de base) pode ser expressa através de redução de risco absoluto, redução de risco relativo e número necessário tratar (quadro I).

A redução de risco relativo é percepcionada como maior e é mais persuasiva que a redução de risco absoluto ou o número necessário tratar, sugerindo benefícios superiores aos que realmente existem. ${ }^{1,8}$ Além disso, as reduções de risco relativo ou absoluto são melhor compreendidas do que o número necessário tratar. No entanto, não é certo que maior compreensão, percepção ou persuasão se traduzam em diferenças no comportamento de adesão a testes, rastreios ou tratamentos. ${ }^{8}$ Curiosamente, a revisão sugere que os profissio-

\begin{tabular}{|l|l|c|}
\hline $\begin{array}{l}\text { QUADRO I. A redução do risco de morte por CCR na forma de redução de risco absoluto, risco relativo ou número } \\
\text { necessário tratar }\end{array}$ & \begin{tabular}{c}
\multicolumn{1}{|c|}{ Definição } \\
PSOF reduz o número de pessoas que morrem \\
de CCR de 8 em 1.000 para 7 em 1.000
\end{tabular} \\
\hline Redução de risco absoluto & $\begin{array}{l}\text { Medida de eficácia de tratamento em termos } \\
\text { de número absoluto de pessoas salvas }\end{array}$ & \multicolumn{1}{|c|}{$0.1 \%$} \\
\hline Redução de risco relativo & $\begin{array}{l}\text { Medida de eficácia de tratamento em termos } \\
\text { de número relativo de pessoas salvas }\end{array}$ & $12,5 \%$ \\
\hline Número necessário tratar & $\begin{array}{l}\text { Número de pessoas que necessitam de } \\
\text { participar num tratamento para se salvar } \\
\text { uma vida. Quanto menor o NNT, melhor } \\
\text { o tratamento. }\end{array}$ & 1.000 \\
\hline
\end{tabular}


nais de saúde apresentam dificuldades semelhantes aos outros participantes na interpretação dos diferentes formatos numéricos. ${ }^{8}$

Para organizações que pretendem promover uma determinada intervenção (como é o caso de serviços de saúde pública, de sociedades científicas e da indústria farmacêutica), a redução de risco relativo é a melhor forma de apresentar informação, dado ser mais persuasiva. Contudo, do ponto de vista dos utentes, o melhor formato será aquele que resulte em decisões mais consistentes com uma boa compreensão e com os seus valores e preferências pessoais. ${ }^{8}$ Se a redução de risco relativo for utilizada deve ser igualmente apresentado o risco de base ou a variação absoluta de risco. De modo contrário, a redução de risco relativo poderá conduzir a decisões mal informadas, particularmente quando o risco de base for reduzido. Por exemplo, o rastreio por PSOF associa-se a uma redução relativa do risco de morrer por CCR de $12,5 \%$. No entanto, quando se transmite também que o risco de morte por CCR é $0,8 \%$ tem--se uma percepção de menor efeito do rastreio. De qualquer forma, a magnitude da variação de risco é melhor transmitida sob a forma de redução de risco absoluto $(0,1 \%)$, principalmente se apresentada como frequência natural (1 em 1.000). ${ }^{8}$

Em relação à transmissão da informação numérica, a prova científica é escassa sobre as diferenças entre a comunicação verbal e a comunicação escrita. A maioria da investigação tem-se centrado na comunicação escrita. Com efeito, isto suscita algumas questões, dado que a maioria dos profissionais de saúde providencia informações verbalmente. ${ }^{2}$

Em nosso entender, a importância do formato numérico na comunicação de risco levanta a necessidade da educação médica abordar as vantagens e desvantagens dos diferentes formatos numéricos, privilegiando as frequências naturais e a redução de risco absoluto. A adopção destes formatos conduzirá a uma melhor compreensão e a uma percepção mais realista da informação. Além disso, torna-se impossível memorizar os riscos associados a todas as doenças e intervenções com as quais o médico de família se pode deparar. É essencial aumentar a acessibilidade à informação e a materiais de apoio à consulta.

\section{Gráficos}

O Sr. Silva ainda não se sentia suficientemente escla- recido para tomar uma decisão sobre o rastreio. A Dra Prudência pesquisou então na Internet uma série de folhetos com gráficos sobre os benefícios e prejuízos do rastreio por PSOF. Mas quais são os melhores gráficos para esclarecer o Sr. Silva?

O uso de gráficos é recomendado para complementar a informação verbal e numérica. ${ }^{9-10}$ De facto, a conversão do risco numérico em gráficos pode facilitar a percepção visual da informação do risco. ${ }^{9,11} \mathrm{O}$ melhor desenho para um gráfico depende do objectivo da comunicação do risco: promover a compreensão de dados quantitativos ou mudar comportamentos. ${ }^{11}$ Para isso, podem ser utilizados diferentes tipos de gráficos, como os gráficos de ícones, os gráficos de barras e circulares, as escadas e escalas de risco e as curvas de sobrevivência e mortalidade.

\section{Gráficos de ícones}

Os gráficos de ícones (figura 1) permitem uma representação visual do risco através de um conjunto de figuras, em que cada figura representa uma pessoa ou grupo (variável discreta). Os dados são melhor compreendidos nesta forma do que através de probabilidades ou percentagens. ${ }^{11}$ Diversos estudos sugerem que os pictogramas exprimem melhor o risco em relação a outros tipos de gráfico. ${ }^{12-14} \mathrm{~A}$ analogia visual com a informação de risco é clara para pessoas com baixo grau de numeracia e mostra a relação da parte com o todo. ${ }^{2}$

\section{Gráficos de barras}

Os gráficos de barras são amplamente conhecidos, mas parece que não facilitam a percepção da informação de um ponto de vista personalizado, relevante para o próprio. ${ }^{2}$ Adicionalmente, as características gráficas que favorecem a compreensão dos aspectos quantitativos diferem das que introduzem mudanças no comportamento. ${ }^{11}$ Os gráficos que traduzem a relação da parte com o todo (figura 2) facilitam a compreensão da razão entre o numerador (número de indivíduos afectados) e o denominador (toda a população em risco). ${ }^{9,11}$ Se reflectirem apenas o numerador é amplificada a percepção do risco, com consequências na tomada de decisão e comportamento (figura 2).

De certo modo, a característica mais importante num gráfico é a simplicidade visual, pois independentemente da precisão dos aspectos quantitativos, os 


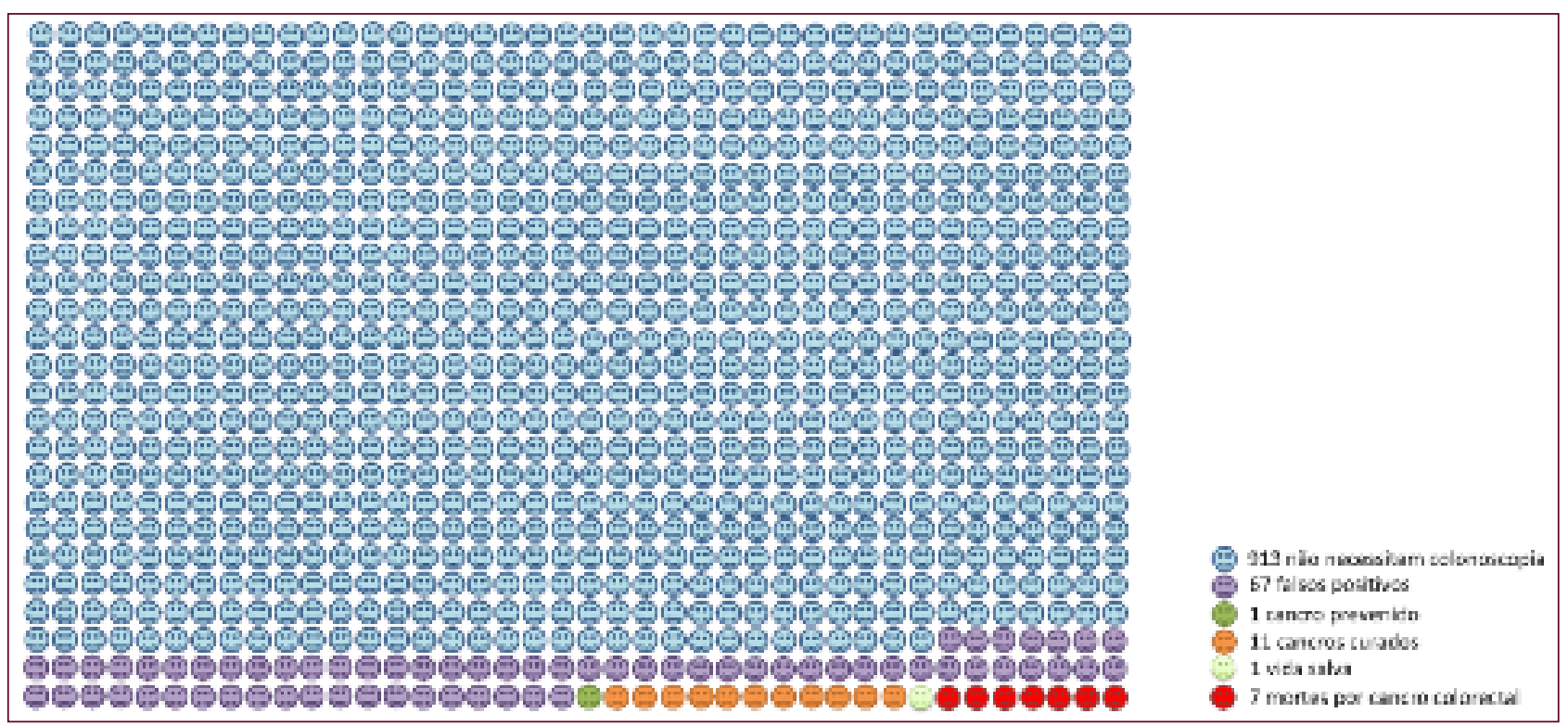

Figura 1. Pictograma. Em 1.000 pessoas submetidas a PSOF, 87 terão de realizar colonoscopia. Em 67 colonoscopias não se vão encontrar alterações (falsos positivos). Das 20 neoplasias ou potenciais neoplasias encontradas, previne-se 1 caso de CCR, 12 neoplasias são curáveis (com a PSOF salva-se 1 vida adicional) e 7 morrerão de CCR.

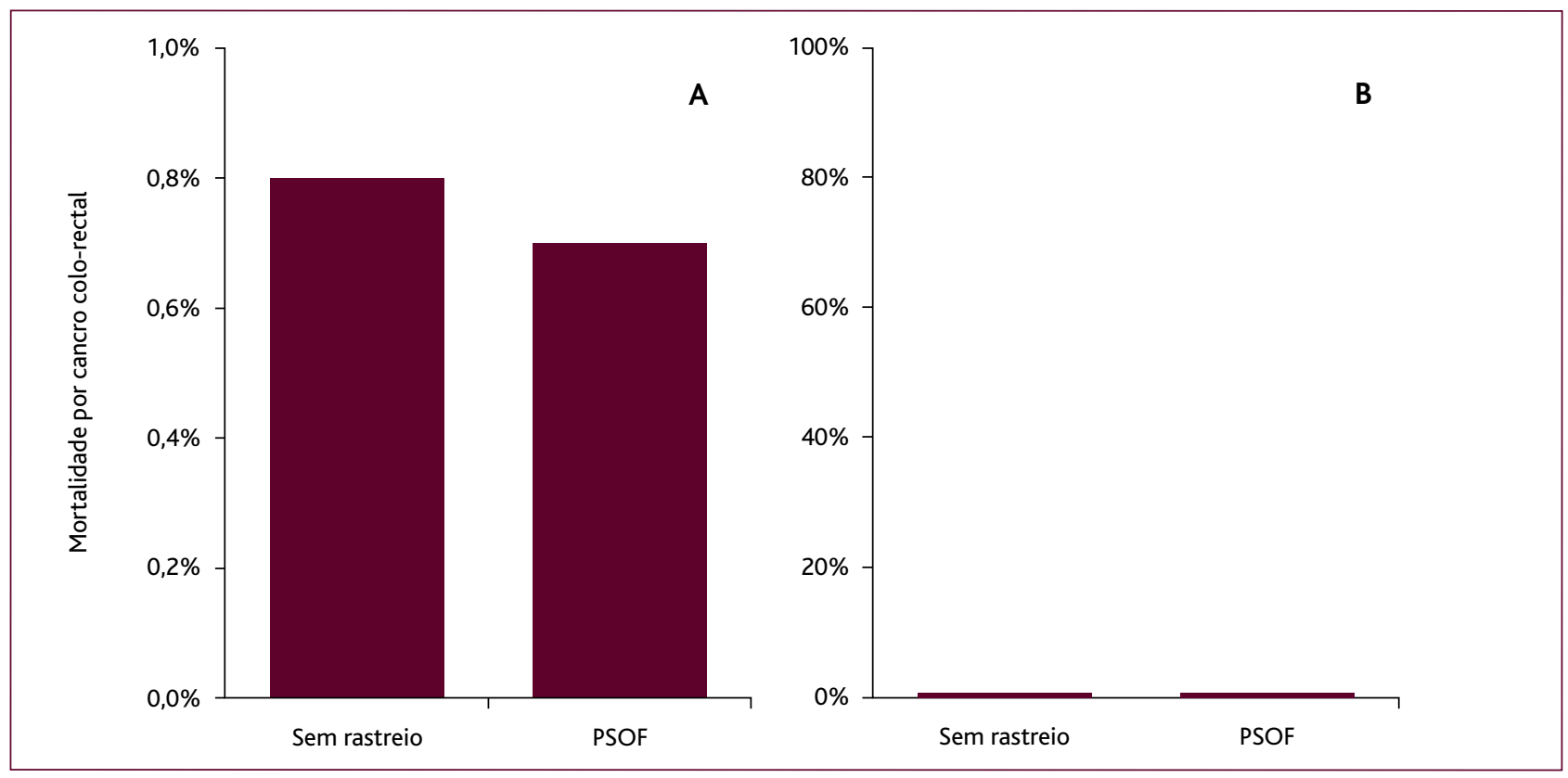

Figura 2. Gráficos de barras. Painel A - Mais persuasivo, porque permite percepcionar a redução de mortalidade com a aplicação do rastreio. Painel B - Mais claro sobre o verdadeiro impacto do rastreio a nível populacional. É muito frequente privilegiar o numerador na comunicacão gráfica. Isto conduz a uma amplificacão da percepção de risco já que a maioria das pessoas não presta atenção à escala da figura.

utentes preferem gráficos com menos elementos visuais. ${ }^{11}$
Tabelas de risco, escadas e escalas de risco

A variação do risco, de muito baixa a muito elevada, 
pode ser apresentada numa tabela vertical (escada) ou horizontal (escala). Um exemplo conhecido deste tipo de gráfico é a tabela de risco SCORE, que resulta da conjugação de vários factores de risco cardiovascular, tomando-se decisões terapêuticas ou preventivas a partir de determinados limiares. Uma das vantagens das escadas ou escalas de risco em relação à comunicação em formato numérico é a capacidade de comparar diversos riscos simultaneamente. ${ }^{11}$ Neste tipo de gráficos é possível introduzir limiares a partir dos quais é necessário tomar determinadas acções.

\section{COMUNICAÇÃO DE RISCO PERSONALIZADA}

A Dra Prudência interroga-se sobre se os riscos que comunicou ao Sr. Silva estarão correctos. Afinal, é um risco calculado a partir de homens e mulheres que participaram em ensaios clínicos realizados noutros países e noutro tempo (há cerca de 20 anos). Talvez fosse melhor procurar estudos que tomassem em consideração o sexo, a idade e outros factores de risco para CCR.

A comunicação personalizada é um diálogo que pretende alcançar uma pessoa em particular, baseando-se nas características que lhe são únicas. A personalização pode tomar em consideração qualquer característica pessoal, nomeadamente factores de risco para uma determinada condição (idade, sexo, antecedentes familiares, hábitos tabágicos, etanólicos, marcadores de risco, entre outros).

A comunicação personalizada deverá basear-se em prova científica de qualidade que permita categorizar o risco de um modo objectivo. Existem estudos que identificam variáveis de prognóstico e desenvolvem modelos matemáticos para prever quem terá maior risco de desenvolver determinada doença. Após estes modelos serem validados em populações diferentes, é possível transformá-los em calculadoras ou outros materiais de consulta. Estas ferramentas devem fornecer, de modo simples e rápido, um risco personalizado, como é o exemplo da ferramenta FRAX ${ }^{\circledR}$. Embora desejável do ponto de vista comunicacional, esta informação de risco personalizada apenas está disponível para um conjunto limitado de doenças. Por esse motivo, a comunicação de risco é geralmente efectuada de um modo pouco sistemático, decorrendo da valorização intuitiva e subjectiva que cada médico atribui aos diferentes factores de risco.
A personalização da informação é apoiada por teoria psicológica e prova empírica. Do ponto de vista teórico, as mensagens personalizadas são mais relevantes para o indivíduo, são melhor processadas e compreendidas. ${ }^{15}$ Consequentemente, as mensagens são mais eficazes a modificar a percepção da susceptibilidade à doença. Segundo o modelo de crenças em saúde, uma determinante-chave dos comportamentos de saúde é a percepção de perigo. ${ }^{16}$ Uma revisão da Cochrane sintetiza a prova empírica: a personalização do risco melhora de modo significativo a tomada de uma decisão informada. Verifica-se um aumento dos conhecimentos referentes aos métodos de rastreio e uma melhor percepção de risco. Em termos de comportamento, parece existir uma maior adesão aos programas de rastreio, principalmente entre os participantes que se consideraram como tendo um risco mais elevado de contrair doença. ${ }^{15}$

\section{INCERTEZA}

A Dra Prudência tem ainda algumas dúvidas em relação aos números que consultou. Por um lado, tranquiliza-a o facto de tratarem-se de dados retirados de uma revisão da Cochrane; mas sabe, por outro lado, que existe sempre risco de viés, os testes usados nos ensaios clínicos são diferentes dos disponíveis nos laboratórios da sua zona e os ensaios tiveram lugar há muitos anos. Além disso, desconhece qual é a população relevante para fornecer o risco de CCR do Sr. Silva: deverá usar os valores da meta-análise (que são antigos) ou estatísticas nacionais (que têm muitos asteriscos e letras pequeninas)? Não se irá enganar nas contas? Com tanta dúvida, o Sr. Silva ainda vai pensar que a Dra Prudência não sabe nada.

É possível diferenciar cinco fontes de incerteza: 1) replicação do risco no futuro; 2) ambiguidade sobre a força/validade da prova científica; 3) significado pessoal de riscos particulares (e.g., gravidade do risco e data de ocorrência do evento); 4) complexidade da informação de risco (multiplicidade de benefícios e prejuízos e incerteza sobre a variação dos mesmos com o tempo); 5) incerteza decorrente da ignorância. ${ }^{17}$

A incerteza sobre uma estimativa de risco é frequentemente expressa através do uso de intervalos de confiança-intervalo de valores que corresponde a uma 
gama de estimativas plausíveis para o verdadeiro risco em função do número de eventos ocorridos. ${ }^{2}$ No entanto, existem fontes de incerteza que não são expressas neste intervalo de valores, podendo conduzir a que se subestime o grau de incerteza.

Por exemplo, quando calculamos o risco para um determinado utente observamos o que se passou em pessoas semelhantes no passado. Essa informação é resumida numa estimativa de risco, assumindo que se aplica ao nosso utente no futuro. Portanto, o risco explica padrões de ocorrência do passado numa população de referência que podem não se replicar. ${ }^{17}$

Em parte, o grau de incerteza depende da força da prova científica. Esta é afectada por diversos factores, nomeadamente: o risco de viés nos estudos individuais; a imprecisão e a inconsistência dos resultados de estudos em populações semelhantes; a correspondência entre as variáveis do estudo e as variáveis de interesse clínico (lesão vascular e doença coronária aguda, por exemplo) e, finalmente, a suspeição de que nem todos os estudos feitos sobre o assunto foram publicados. Qualquer problema nos factores atrás referidos poderá comprometer a confiança nos resultados (aumentar a incerteza). ${ }^{17}$

Além disso, o facto de a avaliação de riscos e o estabelecimento de diagnósticos e de decisões terapêuticas tomarem lugar num encontro clínico breve implica que muitos factos não sejam falados ou apenas sumariamente referidos. Isto pode conduzir a incerteza sobre o estado actual do utente. Os médicos nem sempre revêem os antecedentes familiares e os utentes, por vezes, não se lembram de factores importantes para calcular o risco. ${ }^{17}$

A prova científica é bastante escassa sobre a melhor forma de comunicar a incerteza. Dada a complexidade das respostas cognitivas, emocionais e comportamentais dos utentes, muitos advogam que o foco da comunicação de risco deve ser ajudar os utentes a tolerar e lidar com a incerteza, em vez de ajudar a compreendê- la. $^{18}$

\section{CONCLUSÃO}

A comunicação de risco constitui um pilar importante na promoção de decisões de saúde partilhadas e esclarecidas. $\mathrm{O}$ risco pode ser comunicado sob a forma de números, palavras ou gráficos, sendo que o forma- to escolhido influencia as percepções e os comportamentos do utente. Por exemplo, a personalização da informação e a sua expressão na forma de frequências naturais, risco absoluto e pictogramas favorecem a tomada de uma decisão informada e individualizada. Contudo, independentemente do formato escolhido, é fundamental adequar a mensagem ao utente e tomar em consideração que as decisões resultam da relação que se estabelece entre o médico e o utente.

Felizmente, o Sr. Silva era um utente que a Dr. ${ }^{a}$ Prudência imaginava, enquanto estudava este assunto. $\mathrm{Na}$ próxima vez que tenha um utente que queira falar de rastreio do CCR vai iniciar a conversa com o risco de CCR, usando enquadramento negativo e frequências naturais e vai transmitir os efeitos de tratamento através de riscos absolutos. Entretanto, pediu a um dos alunos do 6. ${ }^{\circ}$ ano que desenhe um pictograma, baseado no quadro resumo da revisão Cochrane. Não vai falar da incerteza numa primeira abordagem.

\section{AGRADECIMENTOS}

Os autores agradecem à Dra. Ana Ribeiro, Dra. Maria João Martins e Dra. Nicole Marques pelos comentários que fizeram a uma versão inicial do manuscrito.

\section{REFERÊNCIAS BIBLIOGRÁFICAS}

1. Gigerenzer G. Reckoning with risk: learning to live with uncertainty. London: Penguin Press; 2003. ISBN 9780140297867

2. French MG. Health literacy and numeracy: workshop summary. Washington, DC: The National Academies Press; 2014. Available from: http://www.nap.edu/catalog.php?record_id=18660

3. Jamoulle M. Prevenção quaternária: a propósito de um desenho. Rev Port Clin Geral. 2012;28(6):398-9. Portuguese

4. Holme $\varnothing$, Bretthauer M, Fretheim A, Odgaard-Jensen J, Hoff G. Flexible sigmoidoscopy versus faecal occult blood testing for colorectal cancer screening in asymptomatic individuals. Cochrane Database Syst Rev. 2013;9:CD009259.

5. Gigerenzer G, Gaissmaier W, Kurz-Milcke E, Schwartz LM, Woloshin S. Helping doctors and patients make sense of health statistics. Psychol Sci Publ Interest. 2008;8(2):53-96.

6. Akl EA, Oxman AD, Herrin J, Vist GE, Terrenato I, Sperati F, et al. Framing of health information messages. Cochrane Database Syst Rev. 2011;12:CD006777.

7. Hoffrage U, Lindsey S, Hertwig R, Gigerenzer G. Medicine: communicating statistical information. Science. 2000;290(5500):2261-2.

8. Akl EA, Oxman AD, Herrin J, Vist GE, Terrenato I, Sperati F, et al. Using alternative statistical formats for presenting risks and risk reductions. Cochrane Database Syst Rev. 2011;3:CD006776.

9. Spiegelhalter $D$, Pearson $M$, Short I.Visualizing uncertainty about the 
future. Science. 2011;333(6048):1393-400.

10. Fischhoff B, Brewer NT, Downs JS. Communicating risks and benefits: an evidence-based user's guide. Silver Spring, MD: The Food and Drug Administration; 2011. Available from: http://www.fda.gov/ScienceResearch/SpecialTopics/RiskCommunication/default.htm

11. Ancker JS, Senathirajah Y, Kukafka R, Starren JB. Design features of graphs in health risk communication: a systematic review. J Am Med Inform Assoc. 2006;13(6):608-18.

12. Hawley ST, Zikmund-Fisher B, Ubel P, Jankovic A, Lucas T, Fagerlin A. The impact of the format of graphical presentation on health-related knowledge and treatment choices. Patient Educ Couns. 2008;73(3):448-55.

13. Lipkus IM. Numeric, verbal, and visual formats of conveying health risks: suggested best practices and future recommendations. Med Decis Making. 2007;27(5):696-713.

14. Fagerlin A, Zikmund-Fisher BJ, Ubel PA. Helping patients decide: ten steps to better risk communication. I Natl Cancer Inst. 2011;103(19):1436-43.

15. Edwards AG, Naik G, Ahmed H, Elwyn GJ, Pickles T, Hood K, et al. Personalised risk communication for informed decision making about taking screening tests. Cochrane Database Syst Rev. 2013;2:CD001865.

16. Becker MH. The health belief model and personal health behaviour. San Francisco: C.B. Slack; 1974.
17. Politi MC, Han PK, Col NF. Communicating the uncertainty of harms and benefits of medical interventions. Med Decis Making. 2007;27(5):681-95.

18. Brashers DE. Communication and uncertainty management. J Commun. 2001;51(3):477-97.

\section{CONFLITOS DE INTERESSE}

Os autores declaram não ter conflito de interesses.

\section{ENDEREÇO PARA CORRESPONDÊNCIA}

Ricardo Rodrigues

USF Conde de Oeiras

Av. Salvador Allende Oeiras

2780-163 Oeiras

E-mail: ricardomoreirarodrigues@gmail.com

Recebido em 08-11-2014

Aceite para publicação em 08-04-2015

André Tomé é editor da RPMGF e não esteve envolvido no processo de revisão editorial do presente artigo.

\section{ABSTRACT}

\section{RISK COMMUNICATION AND RISK PERCEPTION: DIFFERENT WAYS OF COMMUNICATING, DIFFERENT WAYS OF SHARING CLINICAL DECISIONS}

An informed clinical decision arises from a dialogue, where the patient shares biopsychosocial information, preferences and values with his/her physician. Physicians share information about epidemiology and the risk of interventions. We can communicate risk using words, numbers or graphs and the chosen format influences patient's perceptions and behaviour. The format is not always understood, and the inability to reason with numbers (innumeracy) constitutes an important barrier to communicate in an effective way. Despite that, the impact of innumeracy can be lessened through communicational strategies. Frequencies, absolute risk reduction and pictograms convey risk information in a more realistic and understandable way, irrespective of numeracy. There is always uncertainty about risk estimates, but physicians should focus on helping patients dealing with uncertainty. Furthermore, we should understand risk in an individualized context by taking into account personal factors that influence benefits or harms related to the proposed interventions. Regardless of population policies, decisions about health are always made by the patient, who is an autonomous and free person.

Keywords: Risk; Health Communication; Uncertainty; Decision Making; Individualized Medicine. 